\title{
Two-electron distribution functions and short-range electron correlations of atoms and molecules by first principles $T$-matrix calculations
}

\author{
Yoshifumi Noguchi, Soh Ishii, and Kaoru Ohno a) \\ Department of Physics, Graduate School of Engineering, Yokohama National University, 79-5 Tokiwadai, \\ Hodogaya, Yokohama 240-8501, Japan
}

(Received 2 May 2006; accepted 10 August 2006; published online 21 September 2006)

\begin{abstract}
The accurate first principles description of the correlations between electrons has been a topic of interest in molecular physics. We have reported in our previous paper [J. Chem. Phys. 123, 144112 (2005)] that the $T$ matrix, which is the ladder diagrams up to the infinite order, can accurately represent the short-range electron correlations while calculating the double ionization energy spectra of atoms and molecules. In this paper, we calculate the two-electron distribution functions of real systems ( $\mathrm{Ar}, \mathrm{CO}, \mathrm{CO}_{2}$, and $\mathrm{C}_{2} \mathrm{H}_{2}$ ) from the eigenvalue equation associated with the Bethe-Salpeter equation for the $T$ matrix by beginning with the local density approximation of the density functional theory and the $G W$ approximation. We found that when the interelectron distance is very small, the Coulomb hole appears between antiparallel spin electrons due to the short-range repulsive Coulomb interaction. The resulting two-electron distribution functions clearly show the Coulomb hole. () 2006 American Institute of Physics. [DOI: 10.1063/1.2348879]
\end{abstract}

\section{INTRODUCTION}

Two-electron distribution functions provide significant information on the electron correlations between two particles. When the interelectron distance is very small, there are two kinds of electron avoidance known as the "exchange hole" for parallel spins due to Pauli's exclusion principle and the "Coulomb hole" for antiparallel spins due to the shortrange repulsive Coulomb interaction. If the Coulomb hole is described from the first principles, the short-range correlations between electrons can be treated accurately. However, there has been no such theoretical attempt so far because two-particle wave functions are not the simple product of two one-particle wave functions but require a two-particle picture that can effectively include the electron repulsiveness.

In an electron gas, a number of calculations of the radial pair distribution function describing the Coulomb hole have already been performed, and these have indicated the importance of the treatment of short-range electron correlations. For example, the Hartree-Fock approximation fails in evaluating the Coulomb hole, that is, the radial pair distribution function for antiparallel spin electrons is constant over the entire region from the long-range to the short-range limit because of the lack of electron correlations. The random phase approximation (RPA) also fails to evaluate the Coulomb hole. The RPA fails at low densities, i.e., the radial pair distribution function for antiparallel spin electrons becomes negative in the short-range limit. ${ }^{1}$ However, Carbotte ${ }^{2}$ found that the negativeness of the antiparallel spin radial pair distribution function within the RPA can be removed by taking into account the multiple scatterings between electrons in terms of ladder diagrams up to the infinite order referred to as the $T$ matrix; later Hede and Carbotte ${ }^{3}$ and Yasuhara ${ }^{4}$ suc-

${ }^{\text {a)} E l e c t r o n i c ~ m a i l: ~ o h n o @ y n u . a c . j p ~}$ ceeded in calculating the Coulomb hole in an electron gas by evaluating the particle-particle ladder diagrams.

The aim of this paper is to elucidate the effect of the short-range electron correlations between two particles by explicitly calculating the two-electron distribution functions of real systems, for the first time, from the first principles using Green's function method. For this purpose, we introduce a two-particle Green's function and calculate it in the $T$-matrix theory that describes the multiple scatterings between two particles. Then, we solve the Bethe-Salpeter equation (BSE) for the $T$ matrix via an eigenvalue problem and compose the two-electron distribution functions from the eigenfunctions (see next section).

By calculating the double ionization energy (DIE) spectra in which the short-range repulsive Coulomb interaction plays an important role, we have already demonstrated in our previous paper ${ }^{5}$ that the $T$ matrix describes the strong shortrange electron correlations in atoms and molecules. (The calculated DIE spectra are in good agreement with the corresponding experimental values.) The short-range electron correlations cannot be simply expressed by an expectation value of the bare Coulomb interaction $1 / r$. Furthermore, the multiple scatterings between two electrons are very important when the interelectron distance is very small; in the case of the 1,3,5-trithia-2,4,6-triazapentalenyl (TTTA), the effect of the short-range interaction reduces the bare Coulomb interaction by a few $\mathrm{eV}^{6}$

In the present paper, we calculate the two-electron distribution functions of $\mathrm{Ar}, \mathrm{CO}, \mathrm{CO}_{2}$, and $\mathrm{C}_{2} \mathrm{H}_{2}$ by means of the first principles $T$-matrix theory. Deriving the two-electron distribution functions and comparing them with the oneelectron distribution functions, we visually describe the effect of short-range electron correlations in terms of the Coulomb hole. Moreover, we discuss the relationship between 

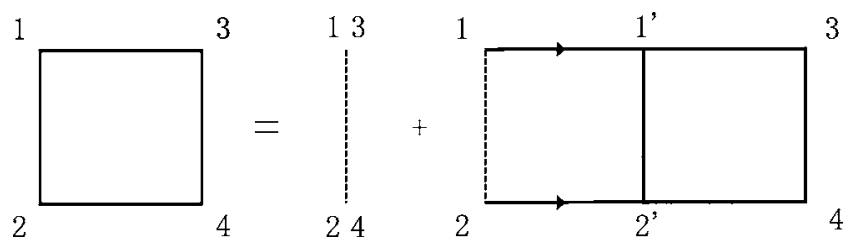

FIG. 1. Schematic representation of the BSE for the $T$ matrix (square). The dotted lines represent the bare Coulomb interaction $U$. The solid lines with an arrow represent the one-particle Green's function $G_{1}$.

the Coulomb hole and short-range electron correlations in detail. The present paper should be regarded as part II of our previous paper. ${ }^{5}$

\section{METHODOLOGY}

First, we begin with local density approximation (LDA) of density functional theory and introduce the $G W$ approximation (GWA) perturbatively. ${ }^{7,8}$ In the present calculation, we employ an all-electron mixed basis approach in which the wave functions are expanded in a linear combination of both atomic orbitals (AOs) and plane waves (PWs). 9,10 This approach can describe all the states from the core states to free electron states efficiently. In order to take into account the short-range Coulomb interactions, we express the twoparticle Green's function $G_{2}$ in terms of the $T$ matrix as follows:

$$
\begin{aligned}
& \int d 3^{\prime} d 4^{\prime} T\left(1,2 \mid 3^{\prime}, 4^{\prime}\right) G_{1}\left(3,3^{\prime}\right) G_{1}\left(4,4^{\prime}\right) \\
& =U(1,2) G_{2}(1,2 \mid 3,4),
\end{aligned}
$$

where $U(1,2)$ is the bare Coulomb interaction and $G_{1}$ is the one-particle Green's function. The $T$ matrix satisfies the following BSE: ${ }^{11}$

$$
\begin{aligned}
T(1,2 \mid 3,4)= & U(1,2) \delta(1-3) \delta(2-4) \\
& +i U(1,2) \int d 1^{\prime} d 2^{\prime} K\left(1,2 \mid 1^{\prime}, 2^{\prime}\right) \\
& \times T\left(1^{\prime}, 2^{\prime} \mid 3,4\right),
\end{aligned}
$$

where $K$ is the noninteracting two-particle Green's function $K\left(1,2 \mid 1^{\prime}, 2^{\prime}\right)=i G_{1}\left(1^{\prime}, 1\right) G_{1}\left(2^{\prime}, 2\right)$. Here, we introduce a shorthand notation $\left(\boldsymbol{r}_{1}, t_{1}\right) \equiv 1$. Diagrammatically, the $T$ matrix is represented in Fig. 1. This equation is sandwiched by the LDA one-particle wave functions and is reduced to a matrix equation. ${ }^{5} K_{\alpha \beta \nu \mu}(\omega)=K_{\nu \mu}(\omega) \delta_{\alpha \nu} \delta_{\beta \mu}$ is then given by

$$
K_{\nu \mu}(\omega)=-\frac{\delta_{\nu}^{\mathrm{occ}} \delta_{\mu}^{\mathrm{occ}}}{\omega-E_{\nu}-E_{\mu}-i \eta}+\frac{\delta_{\nu}^{\mathrm{emp}} \delta_{\mu}^{\mathrm{emp}}}{\omega-E_{\nu}-E_{\mu}+i \eta},
$$

where $E_{\nu}$ and $E_{\mu}$ denote the one-particle energies determined by the GWA and $\delta_{\nu}^{\text {occ }}\left(\delta_{\nu}^{\mathrm{emp}}\right)$ is unity if $\nu$ is occupied (empty) and zero otherwise. We define the two-particle Hamiltonian $H$ as

$$
H_{\alpha \beta \nu \mu} \equiv\left(\frac{f_{\nu \mu}}{K_{\nu \mu}(\omega)}-\omega\right) \delta_{\alpha \nu} \delta_{\beta \mu}-U_{\alpha \beta \nu \mu} f_{\nu \mu}
$$

where we defined $f_{\nu \mu}$ as $f_{\nu \mu}=-\delta_{\nu}^{\mathrm{occ}} \delta_{\mu}^{\mathrm{occ}}+\delta_{\nu}^{\mathrm{emp}} \delta_{\mu}^{\mathrm{emp}}$. Note that the $\omega$ dependence in $K_{\nu \mu}(\omega)$ is eliminated by subtracting $\omega$ in Eq. (4) and thus $H_{\alpha \beta \nu \mu}$ is independent of $\omega$. Then, we solve the eigenvalue problem as follows:

$$
\sum_{\nu \mu} H_{\alpha \beta \nu \mu} A_{\nu \mu}(\Omega)=\Omega A_{\alpha \beta}(\Omega) .
$$

From the eigenvalues $\Omega$ and eigenfunctions $A_{\nu \mu}(\Omega)$, one can construct the $T$ matrix associated with both the hole-hole and electron-electron two-particle Green's functions. The eigenvalues $\Omega$, which are the poles of the $T$ matrix, directly provide the DIE spectra. $A_{\nu \mu}$ represents the coefficients (in the double expansion using the LDA one-particle wave functions) for the spatial part of the Bethe-Salpeter amplitude (two-particle wave function),

$$
\Psi_{\Omega}\left(\boldsymbol{r}, \boldsymbol{r}^{\prime}\right)=\sum_{\nu \mu} A_{\nu \mu}^{*}(\Omega) \psi_{\nu}(\boldsymbol{r}) \psi_{\mu}\left(\boldsymbol{r}^{\prime}\right),
$$

which is either symmetric or antisymmetric with respect to the interchange of the particles. The absolute square of Eq. (6) represents the two-electron distribution function for a given two-particle state $\Omega$.

For the calculation with regard to the $\mathrm{CO}_{2}$ molecule, we employ an fcc supercell with a cubic edge of 30 a.u. and the corresponding PW cutoff energy is $25.1 \mathrm{Ry}$. In the calculation of $\Sigma_{x}$ and $U$, the cutoff energy of the $\mathbf{G}$ vectors is 46.0 Ry. For the calculation of $\Sigma_{c}$, the cutoff energy of $\mathbf{G}$ $\left(\mathbf{G}^{\prime}\right)$ is $19.5 \mathrm{Ry}$ and 5000 levels $(462 \mathrm{eV})$ are needed to achieve a good convergence of the results. The details of the calculation for other atoms and molecules are the same as in Ref. 5.

\section{RESULTS AND DISCUSSION}

Our approach, through which the eigenvalue problem [Eq. (5)] is solved, can be used to calculate the two-electron distribution functions from Eq. (6). The Bethe-Salpeter amplitude (two-particle wave function) $\Psi_{\Omega}\left(\boldsymbol{r}, \boldsymbol{r}^{\prime}\right)$ is in a double space with coordinates $\boldsymbol{r}$ and $\boldsymbol{r}^{\prime}$ that refer to the positions of electrons. If the eigenfunctions $A_{\nu \mu}(\Omega)$ are replaced by $\delta_{\nu \mu}$, $\Psi_{\Omega}\left(\boldsymbol{r}, \boldsymbol{r}^{\prime}\right)$ will not include any electron correlation between the two particles, i.e., we have $\Psi_{\Omega}\left(\boldsymbol{r}, \boldsymbol{r}^{\prime}\right)$ without the interaction. To discuss $\Psi_{\Omega}\left(\boldsymbol{r}, \boldsymbol{r}^{\prime}\right)$ with the interaction, we fix the position of one electron at $\boldsymbol{r}^{\prime}$ and consider the distribution of the other electron as the function of $\boldsymbol{r}$. In this section, the effect of the short-range electron correlation and the Coulomb hole is discussed in detail.

\section{A. Argon}

First, we solve Eq. (5) for an isolated argon atom. Figure 2 shows the absolute square of the two-particle wave functions $\left|\Psi_{\Omega}\left(\boldsymbol{r}, \boldsymbol{r}^{\prime}\right)\right|^{2}$ without (dotted lines) and with (solid lines) the interaction. This two-particle state corresponds to the $3 p$ valence level in the viewpoint of one-particle picture. Here, an atomic nucleus is the origin of the horizontal axis, and an electron is fixed at the position $\boldsymbol{r}^{\prime}=(\mathrm{a}),-2$, (b) -9 , (c) -19 , and (d) -44 on the horizontal axis. The scale of the horizontal axis is indicated in Fig. 2(a). The dotted lines are identical to the absolute square of the one-particle wave functions 


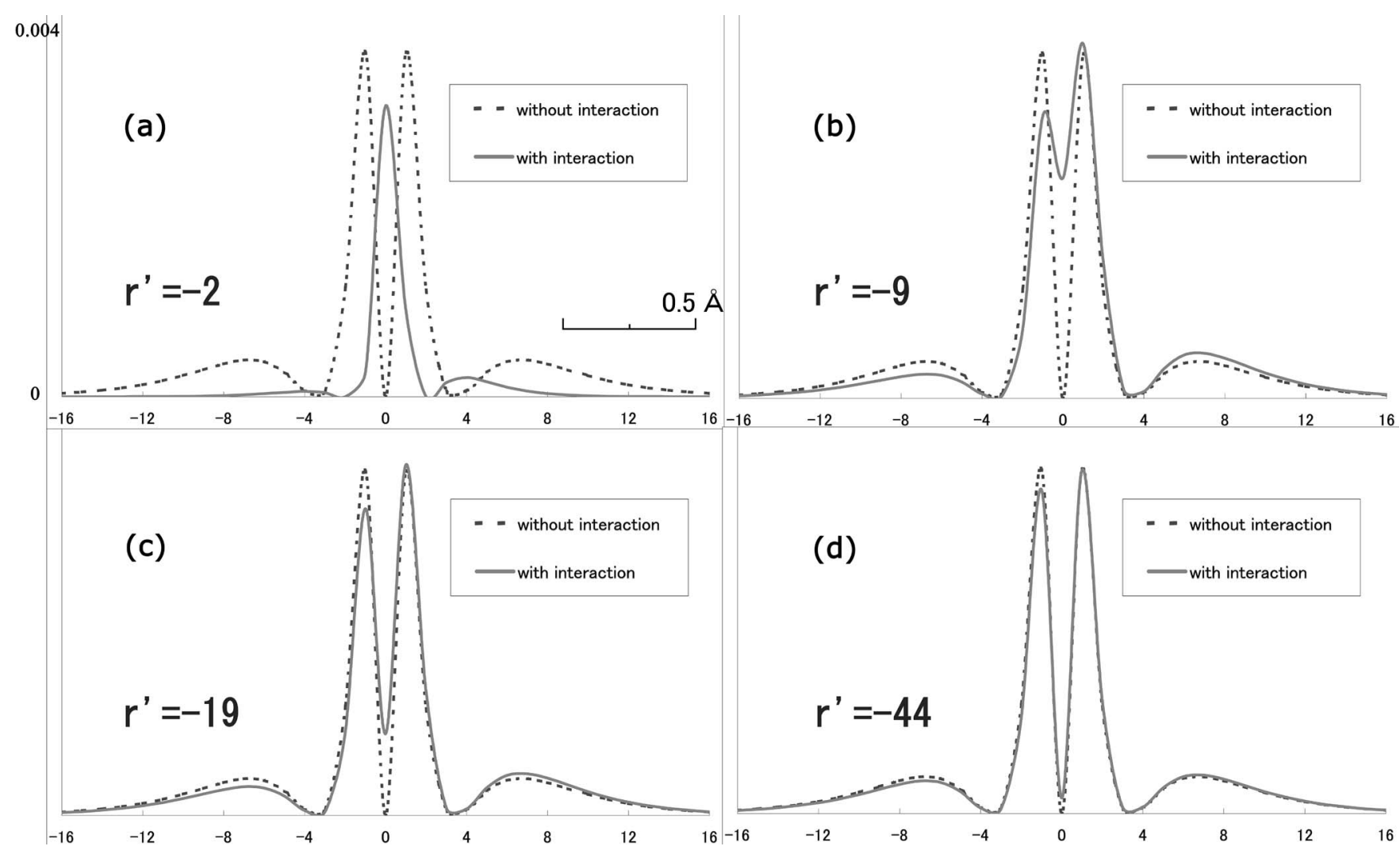

FIG. 2. The two-electron distribution functions without (dotted lines) and with (solid lines) interaction of Ar. Here, an atomic nucleus is the origin of the horizontal axis given in mesh unit ( 1 mesh is about $0.08 \AA$ ) and one electron is assumed to fix at $\boldsymbol{r}^{\prime}=-2$ (a), -9 (b), -19 (c), and -44 (d) on the horizontal axis, respectively. The scale of the horizontal axis is indicated in (a).

$\left|\psi_{\nu}(\boldsymbol{r})\right|^{2}(\nu=3 p)$, which do not include the electron correlations between two particles and have a point symmetry at the origin of the horizontal axis (and no amplitude at the origin). Needless to say, the dotted lines do not change according to the position of the fixed electron. On the other hand, the solid lines include the effect of the short-range electron correlations (i.e., multiple scattering) via the $T$-matrix theory. Because there is a strong repulsive Coulomb interaction from the fixed electron, the shapes of the four solid lines in Fig. 2 are different from those of the dotted lines. The remarkable common characteristics of the solid lines in Figs. 2(a)-2(d) are the breaking of the point symmetry, and a finite amplitude at the origin, although this state is nevertheless the $3 p$ level. The repulsive Coulomb interactions have a stronger effect when the fixed electron is closer to the atomic nucleus; therefore, the change in shape from the dotted lines is the largest in Fig. 2(a) and the smallest in Fig. 2(d) among Figs. 2(a)-2(d). In the case of Fig. 2(a), one electron is fixed at the position $\left(\boldsymbol{r}^{\prime}=-2\right)$ where the dotted lines have the maximum amplitude, and an $s$-type character newly appears to screen the atomic nucleus in addition to the electrons that have already distributed around the atomic nucleus. Moreover, there is clearly a change in the electron population over the broad region (not only in the negative region but also the positive region of the horizontal axis except at the position of the atomic nucleus). Next, we fix one electron at the outer position $\left(\boldsymbol{r}^{\prime}=-9\right.$ on the horizontal axis). The solid line in Fig. 2(b) is closer to the dotted line in contrast to the case of Fig. 2(a), although there still remains the $s$-type character and the breaking of the point symmetry. Another remarkable point is that electron depletion appears only in the negative region of the horizontal axis, while the electron distribution in the positive region is slightly higher than that around the dotted line. Because the repulsive Coulomb interaction from the fixed electron is sufficiently screened by the other electron with the same $p$-type character or the electrons with the $s$-type character at the origin, there is no decrease in the electron distribution in the positive region. When an electron is fixed further away from the atomic nucleus, the shapes of the solid and dotted lines resemble each other [see Figs. 2(c) and 2(d)]. In particular, the difference between the solid and dotted lines in Fig. 2(d) is small.

The two-electron distribution function shown in Figs. 2(a) -2 (d) corresponds to the state characterized as ${ }^{1} \Sigma$, which has a DIE of $\Omega=47.8 \mathrm{eV}$ (this value is already given in Ref. 5). Then, the actual Coulomb interaction given by Ref. 12 $\Omega+E_{\nu}+E_{\mu} \quad(\nu=\mu=3 p)$, which acts between two particles with antiparallel spin in the same $3 p$ level and includes the short-range correlations (i.e., multiple scatterings) via the $T$-matrix theory, is estimated to be about $16.0 \mathrm{eV}$. This value is slightly different as compared to the simple expectation value of the bare Coulomb interaction $U_{\nu \mu \nu \mu}(=16.4 \mathrm{eV})$. This decrease of about $0.4 \mathrm{eV}$ by the multiple scatterings is rather small compared to the other molecules discussed below, but it clearly indicates the effect of the short-range correlations, i.e., the Coulomb hole. 


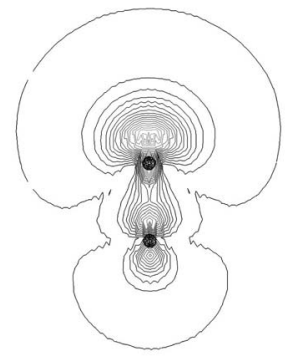

(a)

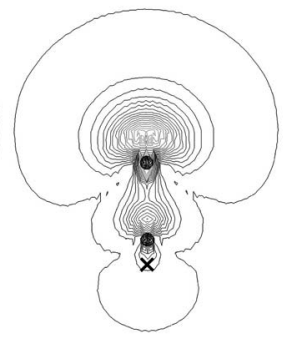

(b)

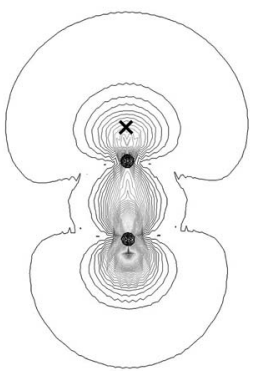

(c)
FIG. 3. The two-electron distribution functions of CO molecule (a) without and [(b) and (c)] with Coulomb interaction. The contour lines are plotted on the molecular plane. Two closed circles are a carbon atom and an oxygen atom from up to down. In (b) and (c), one electron is fixed at the point marked by the cross. The contour lines in (b) and (c) clearly indicate the Coulomb hole around the cross.

\section{B. $\mathrm{CO}$}

We performed a similar calculation for the $\mathrm{CO}$ molecule. Figure 3 shows the calculated two-electron distribution functions (a) without and with [(b), and (c)] the Coulomb interaction in the highest occupied molecular orbital (HOMO) level. Contour lines are plotted on a plane including the molecular axis, and the two closed circles present a carbon atom and an oxygen atom from up to down, respectively. One electron is fixed at both the point marked by the cross [Fig. $3(\mathrm{~b})]$ near the oxygen atom and that [Fig. 3(c)] near the carbon atom. In Figs. 3(b) and 3(c), the electron distribution around the cross is considerably decreased as compared to Fig. 3(a) due to the strong short-range Coulomb interaction from the fixed electron, indicating the Coulomb hole. Another remarkable feature in Figs. 3(b) and 3(c) is that the Coulomb hole moves according to the position in which one electron is fixed. In particular, a considerable enhancement in the electron distribution at the opposite side of the fixed electron is observed in Fig. 3(c).

Comparing Figs. 3(a) and 3(b), we find a slight shift in the electron distribution from the oxygen atom to the carbon atom and an enhancement of the electron distribution around the carbon atom, and identify the Coulomb hole around the cross (near the oxygen atom). Similarly, Fig. 3(c) has a considerable shift in the electron distribution from the carbon atom to the oxygen atom and indicates the Coulomb hole around the cross (near the carbon atom). The electron at the HOMO level of the CO molecule originally has higher distributions in the carbon atom side than the oxygen atom side. Therefore, a shift in the electron distribution from the oxygen atom to the carbon atom is not significant even if one electron is fixed near the oxygen atom. These results are physically reasonable and correctly show the effect of the strong short-range electron correlations.

Here, using the $T$-matrix theory, we estimate the actual Coulomb interaction between the electrons $\Omega+E_{\nu}+E_{\mu}$ to be about $13.0 \mathrm{eV}$, while the corresponding bare Coulomb interaction $U_{\nu \mu \nu \mu}(\nu=\mu=\mathrm{HOMO})$ is about $15.0 \mathrm{eV}$. This difference of $2.0 \mathrm{eV}$ is due to the Coulomb hole. To discuss the Coulomb hole, the short-range electron correlations have to be accurately treated as in the present study. The short-range electron correlations obtained from the evaluation of the lad-

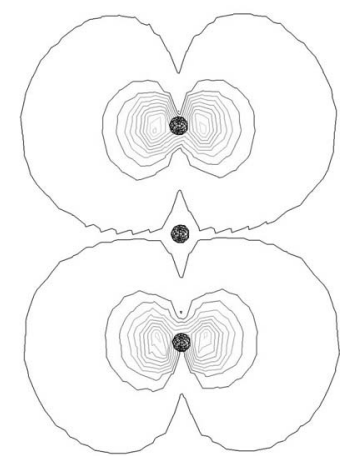

(a)

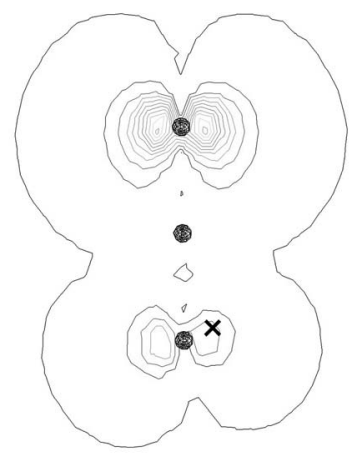

(b)
FIG. 4. The two-electron distribution functions of $\mathrm{CO}_{2}$ molecule without (a) and with (b) interaction. The center closed circle is a carbon atom and the others are oxygen atoms. In (b), the one electron is fixed at the point marked by the cross.

der diagrams up to the infinite order correctly include the effect of the multiple scattering between two particles.

\section{C. $\mathrm{CO}_{2}$}

We plot the two-electron distribution functions of the $\mathrm{CO}_{2}$ molecule in the HOMO level on the molecular plane as shown in Fig. 4. The closed circle at the center in Fig. 4 is a carbon atom and the others are oxygen atoms. The twoelectron distribution function without the interaction is shown in Fig. 4(a). It has two mirror symmetries, up and down symmetries and right and left symmetries. Furthermore, the mirror axes are nodes with no amplitude. On the other hand, the two-electron distribution function with the interaction, which has one electron fixed at the point marked by the cross, is represented in Fig. 4(b). The node between the up and down sides disappears in Fig. 4(b) due to the effect of the fixed electron; the symmetry such as that in Fig. 4(a) is not observed. We see again the remarkable effect of short-range electron correlations as the Coulomb hole in Fig. 4(b).

The two-electron distribution function discussed here is characterized as ${ }^{1} \Delta$. The corresponding DIE $\Omega$ is calculated to be about $38.4 \mathrm{eV}$ and is in good agreement with the experimental value $(38.5 \mathrm{eV}){ }^{13}$ We also estimate that the actual Coulomb interaction between two particles in the HOMO level is $11.4 \mathrm{eV}$ and the corresponding bare Coulomb interaction is $13.5 \mathrm{eV}$. Therefore, the multiple scatterings between electrons reduce the repulsive Coulomb interaction by $1.9 \mathrm{eV}$, thus reflecting the effect of the Coulomb hole.

To discuss the short-range electron correlations in greater detail, we now present the DIE spectra of the $\mathrm{CO}_{2}$ molecule. Table I shows the calculated DIEs and the corresponding experiments. ${ }^{13,14}$ The DIEs shown in the column of "GWA $\times 2$ " are $-E_{\nu}-E_{\mu}$ obtained from the evaluation of the noninteracting two-particle Green's function $K_{\nu \mu}$, namely, twice the ionization potential within the GWA. The ionization potential within the GWA is evaluated to be about $13.7 \mathrm{eV}$ and is in good agreement with that of the experiment $(13.8 \mathrm{eV}) .{ }^{15}$ The HOMO level of $\mathrm{CO}_{2}$ is the $\pi$ orbital and is twofold degenerated. When two electrons are removed 
TABLE I. The DIEs of $\mathrm{CO}_{2}$ (in eV). $T$ matrix and $U_{\nu \mu \nu \mu}$ denote the results obtained by the $T$ matrix and the matrix element (of the bare Coulomb interaction) carrying the largest contribution to the double ionization energy, respectively. The available photoelectron-photoelectron coincidence spectroscopy (PEPECO) experimental data are given for comparison.

\begin{tabular}{|c|c|c|c|c|}
\hline & \multicolumn{2}{|c|}{ Calc. } & \multirow[b]{2}{*}{$U_{\nu \mu \nu \mu}$} & \multirow{2}{*}{$\begin{array}{c}\text { Expt. } \\
\text { PEPECO }\end{array}$} \\
\hline & $\mathrm{GWA} \times 2$ & $T$ matrix & & \\
\hline${ }^{3} \Sigma$ & 27.37 & 37.95 & 13.41 & $37.35^{\mathrm{a}}, 37.65 \pm 0.3^{\mathrm{b}}$ \\
\hline${ }^{1} \Delta$ & 27.37 & 38.44 & 13.46 & $38.52^{\mathrm{a}}$ \\
\hline${ }^{1} \Sigma$ & 27.37 & 38.75 & 13.41 & $39.30^{\mathrm{a}}$ \\
\hline${ }^{3} \Pi$ & 31.89 & 41.10 & 12.78 & $41.43^{\mathrm{a}}$ \\
\hline${ }^{1} \Pi$ & 31.89 & 41.52 & 12.78 & $42.30^{\mathrm{a}}$ \\
\hline
\end{tabular}

${ }^{\mathrm{a}}$ Reference 13 .

${ }^{\mathrm{b}}$ Reference 14.

from the HOMO level, the DIEs split into three states, namely, ${ }^{3} \Sigma,{ }^{1} \Delta$, and ${ }^{1} \Sigma$, according to the two-hole states. Nevertheless, GWA $\times 2$ is identical in $\Sigma$ and $\Delta$, and a difference in the spin symmetries of ${ }^{3} \Sigma$ and ${ }^{1} \Sigma$ can be ignored because they do not include the effect of the repulsive Coulomb interaction. In every state given in Table I, the differences between GWA $\times 2$ and the experiments are more than $10 \mathrm{eV}$. On the other hand, the $T$ matrix, which denotes the values evaluated with the $T$-matrix theory, considerably improves GWA $\times 2$ and is in good agreement with that of the experiments. The difference with the experiments is about $0.8 \mathrm{eV}$ or less for all the states shown in Table I.

The column of $U_{\nu \mu \nu \mu}$ denotes the matrix element of the bare Coulomb interaction carrying the largest contribution to the DIEs. On the other hand, the actual Coulomb interaction, the difference between the $T$ matrix $(\Omega)$ and $G W A \times 2$ $\left(-E_{\nu}-E_{\mu}\right)$, which includes the effect of the multiple scatterings, is deviated from $U_{\nu \mu \nu \mu}$ by $1.5-3.4 \mathrm{eV}$.

\section{D. $\mathrm{C}_{2} \mathrm{H}_{2}$}

The two-electron distribution functions without and with the interaction in the $5 \sigma$ level (i.e., HOMO-1) of the $\mathrm{C}_{2} \mathrm{H}_{2}$ molecule are plotted on a molecular plane as shown in Fig. 5. The four closed circles denote hydrogen, carbon, carbon, and hydrogen atoms from top to bottom. To compare the twoelectron distribution function with the interaction shown in Fig. 5(b) with the two-electron distribution function without the interaction shown in Fig. 5(a), we fix one electron at the point marked by the cross (near the hydrogen atom) in Fig. 5(b). A significant decrease in electron distribution around the fixed electron is observed. On the other hand, there is an increase in the electron distribution around the upper carbon atom at the opposite side of the fixed electron. We confirm the Coulomb hole around the fixed electron and the shift in the electron distribution from the $\mathrm{CH}$ of the fixed electron side to the $\mathrm{CH}$ of the opposite side.

The change in the electron distribution is considerable and is caused by the short-range electron correlation that is clearly indicated by the difference with the bare Coulomb interaction $U_{\nu \mu \nu \mu}(=12.8 \mathrm{eV}$ ) (with $\nu=\mu=\mathrm{HOMO}-1$ ). The actual Coulomb interaction $\Omega+E_{\nu}+E_{\mu}$ is about $8.8 \mathrm{eV}$. The

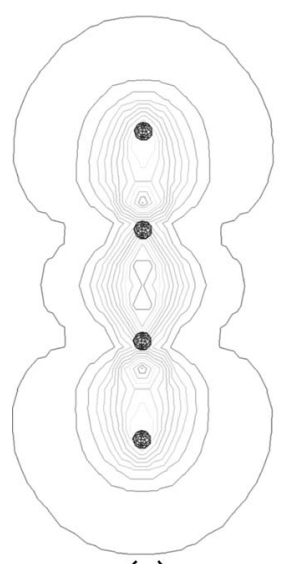

(a)

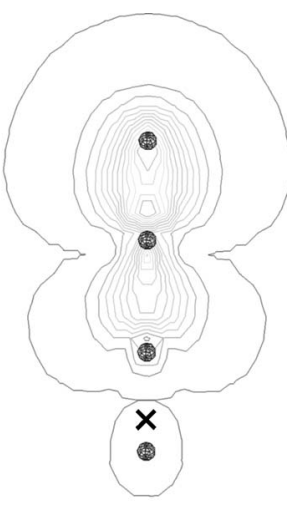

(b)
FIG. 5. The two-electron distribution functions (a) without and (b) with interaction of $\mathrm{C}_{2} \mathrm{H}_{2}$ molecule. The contour lines are plotted on the molecular plane. From top to bottom, the four closed circles denote hydrogen, carbon, carbon, and hydrogen atoms, respectively. In (b), one electron is fixed at the point marked by the cross.

difference with the bare Coulomb interaction $U_{\nu \mu \nu \mu}$ is about $4.0 \mathrm{eV}$. This large effect of the short-range interaction, which reduces the bare Coulomb interaction by about $4.0 \mathrm{eV}$, clearly indicates the existence of the Coulomb hole.

\section{SUMMARY}

In summary, we calculated the two-electron distribution functions and the DIE spectra of $\mathrm{Ar}, \mathrm{CO}, \mathrm{CO}_{2}$, and $\mathrm{C}_{2} \mathrm{H}_{2}$ and discussed the relations between them. We treated the twoparticle Green's function $G_{2}$ by using the first principles $T$-matrix theory to describe the short-range electron correlations. The calculated two-electron distribution functions, which include the multiple scatterings via the evaluation of the ladder diagrams up to the infinite order, clearly indicate the Coulomb hole. Moreover, we explained the relationship between the short-range electron correlations (the difference between $\Omega+E_{\nu}+E_{\mu}$ and $\left.U_{\nu \mu \nu \mu}\right)$ and the Coulomb hole in detail. In the cases of an isolated Ar atom and CO molecule, in particular, we found that the electron distribution is affected by the other electron and may in some cases concentrate on the atomic nucleus to screen its positive charge.

For the $\mathrm{CO}_{2}$ molecule, we presented new results of the first ionization potential and the DIE spectra and compared them with the corresponding experiments as well as our previous study on other molecules. ${ }^{5}$ The calculated first ionization potential and DIEs for the $\mathrm{CO}_{2}$ molecule are in good agreement with the experiments. The short-range electron correlations play an important role in the determination of the double ionization energies. Again, we confirmed that the $T$ matrix well describes the short-range electron correlations between two particles.

\section{ACKNOWLEDGMENTS}

The authors are grateful to the Information Initiative Center, Hokkaido University, for the use of the HITACHI SR8000 and SR11000 supercomputing facilities. This work has been partly supported by Grants-in-Aid of the Scientific Research B (No. 17310067) and Scientific Research on Pri- 
ority Areas (No. 18036005) from Japan Society for the Promotion of Science and from the Ministry of Education, Culture, Sports, Science and Technology, respectively. One of the authors (Y.N.) is supported by the Research Fellowship, Japan Society for the Promotion of Science.

${ }^{1}$ A. Glick and R. A. Ferrell, Ann. Phys. (N.Y.) 11, 359 (1960).

${ }^{2}$ J. P. Carbotte, Can. J. Phys. 18, 837 (1967).

${ }^{3}$ B. B. J. Hede and J. P. Carbotte, Can. J. Phys. 50, 1756 (1972).

${ }^{4}$ H. Yasuhara, Physica (Amsterdam) 78, 420 (1974).

${ }^{5}$ Y. Noguchi, Y. Kudo, S. Ishii, and K. Ohno, J. Chem. Phys. 123, 1441122 (2005)
${ }^{6}$ K. Ohno, Y. Noguchi, T. Yokoi, S. Ishii, J. Takeda, and M. Furuya, ChemPhysChem 7, 1820 (2006).

${ }^{7}$ L. Hedin, Phys. Rev. 139, A796 (1965).

${ }^{8}$ M. S. Hybertsen and S. G. Louie, Phys. Rev. B 34, 5390 (1986).

${ }^{9}$ K. Ohno, F. Mauri, and S. G. Louie, Phys. Rev. B 56, 1009 (1997).

${ }^{10}$ S. Ishii, K. Ohno, V. Kumar, and Y. Kawazoe, Phys. Rev. B 68, 195412 (2003).

${ }^{11}$ M. Springer, F. Aryasetiawan, and K. Karlsson, Phys. Rev. Lett. 80, 2389 (1998).

${ }^{12}$ J. Kanamori, Prog. Theor. Phys. 30, 275 (1963).

${ }^{13}$ J. H. D. Eland, Chem. Phys. 294, 171 (2003).

${ }^{14}$ T. Masuoka, Phys. Rev. A 50, 3886 (1994).

${ }^{15} \mathrm{http} / / /$ webbook.nist.gov/ 